\title{
Stereoscopic acuity and observation distance
}

\author{
Mark F. Bradshaw ${ }^{1}$ \& Andrew Glennerster ${ }^{2}$ \\ ${ }^{1}$ Department of Psychology, \\ School of Human Sciences, \\ University of Surrey, \\ Guildford, \\ GU2 7XH, UK. \\ ${ }^{2}$ University Laboratory of Physiology, \\ Parks Road, \\ Oxford, \\ OX1 3PT, UK.
}

Phone: +44 1865272558

e-mail: ag@physiol.ox.ac.uk

$18 / 3 / 05$ 


\section{Abstract}

The amount of depth perceived from a fixed pattern of horizontal disparities varies with viewing distance. We investigated whether thresholds for discriminating stereoscopic corrugations at a range of spatial frequencies were also affected by viewing distance or whether they were determined solely by the angular disparity in the stimulus prior to scaling. Although thresholds were found to be determined primarily by disparity over a broad range of viewing distances, they were on average a factor of two higher at the shortest viewing distance $(28.5 \mathrm{~cm})$ than at larger viewing distances $(57$ to $450 \mathrm{~cm})$. We found the same pattern of results when subjects' accommodation was arranged to be the same at all viewing distances. The change in thresholds at close distances is in the direction expected if subjects' performance is limited by a minimum perceived depth. 


\section{Introduction}

The magnitude of the horizontal disparities projected by an object in the world depends on both its structure and its distance from the observer. For a real object, the horizontal disparity between any pair of points on an object varies roughly inversely with the square of the viewing distance (Kaufman, 1974). Therefore, to determine absolute depth (and to maintain stereoscopic depth constancy) from horizontal disparity it is necessary to incorporate information about viewing distance. The issue of depth constancy has a long history in the study of visual psychophysics and has recently seen an up-surge in interest (Wallach \& Zuckerman, 1963; Ono \& Comerford, 1977; Foley, 1980; Rogers \& Bradshaw, 1993; Tittle et al, 1995; Glennerster et al, 1996). Estimates of the magnitude of depth scaling accomplished by the visual system range from $30 \%$ to $100 \%$ (e.g. Johnston, 1991; Cumming et al, 1991; Rogers \& Bradshaw, 1993; Rogers \& Bradshaw, 1995; Bradshaw \& Rogers 1996). This difference is mainly accounted for by two experimental factors. The distance cues in the stimulus (e.g. Predebon, 1993; Glennerster et al, 1998) and the task the observer is required to do (Glennerster et al, 1996). Related results in physiology have been reported by Trotter et al (1992) who showed that some cells in area V1 of the monkey modulate their response with changes in viewing distance which suggests that retinal disparity alone may not determine the response characteristics of all the cells underlying disparity processing in the early visual pathway (see also Judge and Cumming, 1986; Erkelens, 2001).

These experiments demonstrate that the visual system takes viewing distance into account when making judgments about depth based on horizontal disparities. The question we focus on in the present paper is whether viewing distance is also taken into account when the sensitivity of the visual system to disparity information is determined - or whether thresholds are solely determined by angular disparity as measured at the retinae. Such an 
idea would at first seem to run counter to the traditional framework for understanding psychophysical thresholds based on signal detection theory (Green and Swets, 1966) which seeks to explain thresholds only in terms of the magnitude of the input signal (in this case disparity) and noise. Nevertheless, the idea of a minimum perceived depth limit is a longstanding one (Jameson \& Hurvich, 1959; Ogle, 1958; see also Cormack, 1984; Harris et al, 1998) and there are several reasons to test the hypothesis again.

Both Jameson \& Hurvich (1959) and Ogle (1958) reported that stereoscopic thresholds were determined solely by stimulus disparity (defined in angular terms) and they were invariant with viewing distance. Ogle (1958), for example, presented a 2 line display (one fixation line and one test) at two viewing distances, 0.5 and 10 metres. Observers were required to judge whether the test line was nearer or farther and he found a negligible effect of viewing distance. There are a number of reasons why the conclusions of these studies may not be definitive. First, the stereo thresholds reported by Ogle (1958) and Jameson \& Hurvich (1959) were at least an order of magnitude higher than typically found with more modern experimental techniques which use rich (random-dot) surfaces as stimuli (e.g. Bradshaw \& Rogers, 1999). Therefore the stereo system in the former experiments may not actually have been at a true threshold. Second, in the studies by both Ogle (1958) and Jameson \& Hurvich (1959), all cues to viewing distance with the exception of vergence angle were eliminated from their displays. Third, the closest viewing distances used by Jameson and Hurvich (1959) was $3 \mathrm{~m}$ whereas that used by Ogle was $0.5 \mathrm{~m}$ which may not have been close enough to reveal any viewing distance dependency. This latter point may need some explanation. Stereoscopic thresholds may be determined by factors before or after viewing distance is taken into account. That is, thresholds may be based on either the minimum amount of depth available in the stimulus, the minimum amount of disparity, or both. 
Crucially, which aspect controls performance may vary with viewing distance as represented in schematic form in figure 1.

\section{Figure 1 about here}

If thresholds were determined only by the disparity in the stimulus, then performance should correspond to the horizontal dotted line (i.e. independent of viewing distance). On the other hand, if performance were determined by a fixed threshold depth, then thresholds plotted in terms of disparity would be small at far viewing distances and large at near viewing distances. They should fall along the rising dotted line which shows threshold disparity proportional to $1 / \mathrm{D}^{2}$. (This is a good approximation when the depth is small compared to the viewing distance, D). The solid curve shows the threshold that would be expected if performance were limited by both disparity and depth. The assumption here is that the two sources of noise are combined independently: the one that is responsible for the disparity threshold, $\sigma_{\mathrm{d}}$, which is independent of viewing distance, and the one responsible for the depth or relief threshold, $\sigma_{\mathrm{r}}$. The total variance due to both noise sources is: $\sigma_{\mathrm{t}}^{2}=\sigma_{\mathrm{d}}^{2}+\sigma_{\mathrm{r}}^{2}$. The solid line in figure 1 shows $\sigma_{t}$ as a function of distance. This means that at near viewing distances more disparity would be required to successfully discriminate a stereoscopic stimulus because the factor that primarily determines threshold changes with viewing distance.

The present experiments were designed to re-examine this issue although, in light of the above discussion, we made several changes to the experimental design compared to previous studies. A judgment based on surfaces (at a range of disparity corrugation frequencies to establish a comprehensive picture of the disparity sensitivity functions) was used to determine sensitivity as opposed to judging depth order of two lines. In addition, the 
stimuli were presented in more naturalistic conditions in which many cues to distance are present (Durgin et al, 1995; Glennerster et al, 1996; 1998; Frisby et al, 1996; Bradshaw et al, 1998; O’Leary \& Wallach, 1980; Predebon, 1993) and the range of viewing distances included a relatively near one $(28.5 \mathrm{~cm})$. We also took care, through the use of artificial pupils, that changes in pupil size and accommodative state, which would normally co-vary with viewing distance, would not also change with viewing distance and thereby cause a change in stereoacuity. Further controls were also used across the course of three experiments reported below.

\section{Methods}

\section{Subjects}

Four experienced psychophysical observers took part in the experiments as detailed in each section. Two observers had 6/5 acuity (MFB, AG), one had 6/6 acuity (RAE). The final observer (BJR) was corrected to normal.

\section{Apparatus}

A Wheatstone stereoscope was used to present the stimuli. Two front-silvered mirrors were mounted at 45 degrees to the lines of sight of both eyes in a purpose built mount. Two Macintosh 12-inch monochrome displays (640 by 480 pixels refreshed at $67 \mathrm{~Hz}$ ) were used to present the stimuli. These were precisely positioned so that the vergence angle and vertical disparities were consistent with the observation distance. The luminance of the bright dots was $68 \mathrm{~cd} / \mathrm{m}^{2}$ and the background was $0.74 \mathrm{~cd} / \mathrm{m}^{2}$ measured through the mirrors. Pixel size was $2 \operatorname{arc} \min$ at $57 \mathrm{~cm}$. 
In experiments 1 and 3, thresholds for discriminating the structure in stereoscopic corrugations were established at three different viewing distances. The distance of the eyes from the monitors was either $28.5,57$, or $114 \mathrm{~cm}$. The monitors were positioned on a textured ground plane surface (the texture was accurately matched on either side of the midline which give rise to a single surface when fused), so there were many natural cues for viewing distance.

In experiment 2 , changes in viewing distance were simulated by changing the convergence angle and vertical disparity information. This was accomplished by moving the random dot patch laterally by precise amounts in equal and opposite directions on the display screens. This effectively changed the angle with which each eye viewed the stimulus and changed both the vergence angle and the vertical disparities information for distance (see also Bradshaw et al, 1996). The screens remained at $57 \mathrm{~cm}$ and hence the accommodative demand was the same for all the simulated distances. In this experiment the stimuli were completely masked by matte black cloth which eliminated most (contradictory) cues for viewing distance. The simulated distances were $28.5,57,114,228$ and $456 \mathrm{~cm}$.

In all three experiments, the stimuli were viewed through artificial pupils $(2 \mathrm{~mm}$ in diameter) placed immediately in front of the subjects' eyes. These were carefully positioned at the beginning of each condition so that the centre of the pupil looked directly at the centre of the stimulus. The purpose of these pupils was to minimise any effects of changes in pupil size and to mitigate any changes in accommodation error, optical aberrations and the potential effects vergence jitter.

\section{Stimuli}

The stimuli were $50 \%$ density random dot patterns visible within a circular aperture of 8 deg diameter. Dot size was 4 min arc. Horizontal corrugations sinusoidally modulated in depth were defined by binocular disparity. The spatial frequency and amplitude of the 
modulations were variable and are given below. The vertical centre of each stimulus was marked by two horizontal white lines, $40 \mathrm{~min}$ arc long, superimposed at the left and right edges of the pattern. The pattern of random dots was changed on every trial.

\section{Figure 2 about here}

In experiments 1 and 3, which used different physical viewing distances $(28.5,57$ and $114 \mathrm{~cm}$ ) the angular size of the stimuli was kept constant (see figure 2). Therefore at $28.5 \mathrm{~cm}$ the stimulus was defined by dots of size 1 by 1 pixels within a circular aperture of 120 pixels. At $57 \mathrm{~cm}$, the dot size was 2 by 2 pixels and the diameter of the aperture was 240 pixels; at $114 \mathrm{~cm}$, dot size was 4 by 4 pixels and the diameter of the aperture was 480 pixels. In experiment 1 , the spatial frequency of the depth corrugations was manipulated and ranged from $0.2 \mathrm{cpd}$ to $0.8 \mathrm{cpd}$ in $\sqrt{2}$ steps. To maintain the same spatial frequency, in cpd, at the different viewing distances the modulation was halved as the distance was doubled (see figure 2). Therefore the stimuli at the three viewing distances were all equivalent at the eye in angular terms.

In experiment 2, different viewing distances between 28.5 and $456 \mathrm{~cm}$ were simulated using the method described above. The physical distance to the screens was always $57 \mathrm{~cm}$ and the stimulus characteristics were therefore those described for this distance ( 2 by 2 pixels within an aperture of 240 pixels diameter).

\section{Generation of binocular disparity}

Three separate video cards, resident in an Apple Macintosh IIfx computer were required to create the experimental stimuli. Two of the cards stored identical random-dot patterns and supplied the video signal for the left and right eyes. The third acted as a master and supplied line and sync pulses for one of the other cards. This was necessary as binocular

disparity was created by phase shifting the video signal on one of the video cards. The phase 
shifting technique was chosen as it permitted sub-pixel displacement to be achieved without recourse to software anti-aliasing which may have compromised projected image quality at the different viewing distances.

The clocking signal of the three video cards was provided by a single external 30 $\mathrm{MHz}$ crystal. The two cards (master and slave) involved in disparity generation were completely synchronised. The clock signal of the master card was supplied by a $30 \mathrm{MHz}$ crystal. The clock signal on the slave card was supplied by a Wavetek 178 device $(50 \mathrm{MHz}$ programmable waveform synthesizer) which was set to run at $30 \mathrm{MHz}$ also. The Wavetek 178 was phase locked with the crystal. The Wavetek 178 permitted control of the phase of the clocking signal, relative to the line sync pulse, which means that each line of the video signal from the slave card could be advanced or retarded by precise, sub-pixel, amounts. The amount of distortion applied to each line was determined by a Wavetek 175 device (arbitrary waveform generator) synchronised to the frame rate of the displays (using the frame sync pulse from the master card). By varying the spatial frequency and/or the amplitude of the waveform specified by the Wavetek 175 corrugations with different depth profiles could be displayed. The distortion was applied to the right eye only.

\section{Psychophysical technique}

The Method of Constant Stimuli was used to determine thresholds for detecting the depth corrugations. The observer's task was to report whether the horizontal corrugation lying across the centre of the dot pattern, (marked by the two white lines) was concave (a trough) or convex (a peak). On each trial, the amplitude and phase of the depth signal (disparity and/or motion parallax) was randomly chosen from seven possible values corresponding to $-3,-2,-1,0,1,2$ or 3 times the smallest step size - where negative amplitudes indicate the sinusoidal modulation was in opposite phase. An experimental session consisted of 280 trials (in 4 blocks), corresponding to 40 trials of each of the stimulus 
levels. Frequency of seeing plots were generated from each data set and the best-fitting cumulative Gaussian curve was determined using the probit technique (Finney, 1971). The $75 \%$ correct point on the psychometric function was taken as the threshold value.

\section{Experiment 1: changes in real viewing distance.}

In experiment 1, thresholds for discriminating the structure in stereoscopic corrugations at a range of corrugation frequencies $(0.2$ to $0.8 \mathrm{cpd})$ at 3 different physical viewing distances $(28.5,57$, or $114 \mathrm{~cm})$ were determined. Note, the different corrugation frequencies were used and presented to give a rich description of the effects of viewing distance for surfaces defined by binocular disparity. No interaction between corrugation frequency and viewing distances was expected.

Thresholds at the three viewing distances for each corrugation frequency are shown in figure 3 for one subject (MFB). Figure 3a plots the thresholds in terms of depth (in $\mathrm{mm}$ ) and figure $3 \mathrm{~b}$ plots thresholds against disparity (arc sec).

\section{Figure 3 about here}

If disparity alone determined thresholds then, due to the inverse square law which relates disparity and distance, the plots in figure 3 a should be separated by factors of 4 . If we assume that the average threshold at $114 \mathrm{~cm}$ was $0.4 \mathrm{~mm}$ (top dotted line in figure 3a) then, based on this estimate, the threshold at 57 and $28.5 \mathrm{~cm}$ should be 0.1 and $0.025 \mathrm{~mm}$ respectively (middle and bottom dotted lines respectively). It can be seen in the figure that the observer is more sensitive than predicted at $57 \mathrm{~cm}$ and much less sensitive than predicted at the nearest viewing distance. The same result is clear when the data is replotted against disparity (figure $3 b$ ). The lowest thresholds were determined for the $57 \mathrm{~cm}$ viewing distance across the spatial frequency range and were considerably higher at $28.5 \mathrm{~cm}$. If thresholds 
were determined by disparity alone then the data for each viewing distance should have coalesced. From figure 3, however, it is apparent that thresholds are somewhat higher at near viewing distances. Peak sensitivity for each viewing distance occurred at the same $(0.4 \mathrm{cpd})$ corrugation frequency (see also Bradshaw and Rogers, 1999). Given our intention was to investigate performance as near threshold as possible, the data from $0.4 \mathrm{cpd}$ is shown for all 4 observers as a function of viewing distance in figure 4.

\section{Figure 4 about here}

Figure 4 is plotted in the same form as the schematic presented in figure 1 which illustrated the expected results if there was a separable depth threshold. For each observer, thresholds were higher at $28.5 \mathrm{~cm}$ than they were at 57 or $114 \mathrm{~cm}$. For ease of discussion, and based on the logic of figure 1, we have focused on the increase in threshold between our closest viewing distance, $28.5 \mathrm{~cm}$, and the benchmark distance of $57 \mathrm{~cm}$. Thresholds at 28.5 $\mathrm{cm}$ were significantly higher than those found at $57 \mathrm{~cm}(\mathrm{t}(3)=3.78 ; \mathrm{p}<0.01)^{1}$ when all subjects were considered together. For the 4 subjects, treated separately, change in the threshold values from the psychometric functions can be summarised as a ratio of performance at $28.5 \mathrm{~cm}$ and $57 \mathrm{~cm}: \mathrm{MFB}$, ratio $=1.85(\mathrm{t}(238)=3.13 ; \mathrm{p}<0.01) ; \mathrm{BJR}$, ratio $=$ $1.70\left(\mathrm{t}(238)=5.06 ; \mathrm{p}<10^{-6}\right) ; \mathrm{AG}$, ratio $=1.42(\mathrm{t}(238)=1.93 ; \mathrm{p}<0.05)$ and $\mathrm{RAE}$, ratio $=1.91$ $\left(\mathrm{t}(238)=8.13 ; \mathrm{p}<10^{-12}\right)^{2}$. Of course, given these ratios, a 2-way ANOVA of all 8

\footnotetext{
${ }^{1}$ All significance levels reported are 1-tailed.
}

${ }^{2}$ The degrees of freedom for these t-tests are shown as 238. df for an unpaired t-test is $n_{1}+n_{2}$ -2 , where $n_{1}$ and $n_{2}$ are the number of samples in each group. Although the number of trials in each condition was 280 , simulations show that a probit fit of 280 trials distributed as in our experiment gives a sampling error of the threshold (standard deviation of fitted Gaussian) equivalent to 120 or more random samples of a Gaussian distribution of the same standard deviation. Hence, we have set $n_{1}=n_{2}=120$. 
thresholds shows that the difference between thresholds at $28.5 \mathrm{~cm}$ and $57 \mathrm{~cm}$ is highly significant $\left(\mathrm{F}(1,952)=33.9 ; \mathrm{p}<10^{-7}\right)^{3}$.

Based on the predictions set out above, if thresholds were based solely on angular disparity then the ratios of thresholds at 28.5 and $57 \mathrm{~cm}$ should be 1 (corresponding to horizontal line in figure 1). If the thresholds were based only on depth they should be near 4, but as the predictions shown in figure 1 make clear, any ratio between 1 and 4 is possible according to the model shown, depending on where the 28.5 and $57 \mathrm{~cm}$ points lie relative to the rising part of the curve. In fact, the average the ratio was 1.72. This result suggests that stereoscopic thresholds may not be based solely on the angular retinal disparities in the stimuli but rather that a separate threshold, based on depth, may also be important.

At this stage, however, caution must be exercised when interpreting this result. The key here is to address whether other variables may also have changed with changes in viewing distance. Such variables may have contributed to the observed change in performance and therefore must be considered before any conclusion is reached. Although the small artificial pupils used in the experiment are effective in eliminating changes in pupil size, and hence in reducing accommodative load, some factor may have remained which covaried with changes in viewing distance. Two further experiments, reported below, were

${ }^{3}$ 2-way ANOVA results: subjects and distances $(28.5$ and $57 \mathrm{~cm})$ were treated as two independent parameters. The standard error of the threshold given by probit was known for each 2-way ANOVA cell, allowing the variance ratio, F, to be computed without reference to the number of trials within each cell, $\mathrm{n}$. However, the significance of $\mathrm{F}$ depends on the degrees of freedom for the denominator, which depends on n: d.f. $=n r n c(n-1)$, where $n r$ is the number of distances (2), nc is the number of subjects (4) and $n$ is the number of trials per cell. As explained in the previous footnote, we took 120 as a conservative estimate of $n$. 
therefore designed to test whether other factors, connected to changes in the quality of the images at the eye, may have co-varied with changes in viewing distance.

\section{Experiment 2: simulated changes in viewing distance.}

A potential problem with experiment 1 is the fact that with real, physical changes of viewing distance the optical quality of the projected image may not have been the same at each observation distance - despite the use of stimuli equated in angular terms and artificial pupils. Therefore, the experiment was repeated using simulated observation distances as described in the Methods. Convergence angle and vertical disparity (which were always consistent with the simulated distance) were the main cues used to simulate changes in viewing distance. The range of natural cues for viewing distance which existed in experiment 1 was therefore eliminated or controlled to indicate the actual viewing distance of $57 \mathrm{~cm}$. This technique had the advantage that a wider range of (simulated) viewing distances could be used than was practicable when physical distances were used, and that the physical (accommodative) distance was constant. The same four observers took part as in experiment 1.

\section{Figure 5 about here}

Thresholds for the close viewing distance were again higher than those determined at 57 and $114 \mathrm{~cm}$. Therefore, the main result of experiment 1, that thresholds increased as viewing distance decreased, was replicated. The rise in thresholds between 57 and $28.5 \mathrm{~cm}$ $($ ratio $=1.27)$ was again significant $(\mathrm{t}(3)=3.07 ; \mathrm{p}<0.05)$. For each subject the ratio of thresholds at $28.5 \mathrm{~cm}$ and $57 \mathrm{~cm}$ were: $\mathrm{MFB}$, ratio $=1.26(\mathrm{t}(238)=2.32 ; \mathrm{p}<0.05)$; BJR, ratio $=1.28(\mathrm{t}(238)=2.18 ; \mathrm{p}<0.05) ; \mathrm{AG}$, ratio $=1.29(\mathrm{t}(238)=2.34 ; \mathrm{p}<0.05)$ and RAE, ratio $=$ 
$1.38(\mathrm{t}(238)=2.53 ; \mathrm{p}<0.01)$. Taken together, the thresholds at $28.5 \mathrm{~cm}$ were higher than at $57 \mathrm{~cm}\left(\mathrm{~F}(1,952)=19.5 ; \mathrm{p}<10^{-4}\right)$. Methods of calculating these t-tests and 2-way ANOVA were as in experiment 1 .

The size of the effect of distance is reduced in this experiment compared to experiment 1 , although it is still significant. The reduction may be attributable to the reduced set of distance cues available to signal the viewing distance changes. The principal cue in this case was vergence angle changes, given the size of the stimuli (Bradshaw et al, 1996). Alternatively, the use artificial pupils with fixed accommodative effort at $57 \mathrm{~cm}$ may not have ensured that image quality was similar at all simulated viewing distances. If, for example, the accommodative state of subjects is linked to vergence in these subjects, the changes in vergence could have induced a change in accommodation (and hence a blurred image) despite the constant accommodative demand of a stimulus physically at $57 \mathrm{~cm}$. The purpose of the final experiment was to eliminate any explanation of this kind.

\section{Experiment 3: changes in real viewing distance with constant accommodative effort.}

In this experiment, thresholds were again collected using the 3 physical viewing distances as used in experiment 1. Trial lenses were used so that any accommodative effort normally exerted by a subject in response to the changes in viewing distance was rendered unnecessary — the load exerted by each subject was now approximately the same for each viewing distance and set to $57 \mathrm{~cm}$. That is, the lenses were placed in front of the each eye so that, in order to maintain a well focused image, the observer had to exert the same accommodative effort as that required to focus at $57 \mathrm{~cm}$ without lenses. To accomplish this, +2 diopter lenses 
were used when viewing the stimuli at $28.5 \mathrm{~cm}$ and when viewing the stimuli at $114 \mathrm{~cm}-1$ diopter lenses were used.

Two subjects who previously took part in experiments 1 and 2 took part in this experiment. The results of the experiment are shown in Figure 6. The ratios of thresholds at $28.5 \mathrm{~cm}$ relative to $57 \mathrm{~cm}$ for each subject again indicated a significant rise: for MFB, ratio = $1.33(\mathrm{t}(238)=2.82 ; \mathrm{p}<0.01)$ and $\mathrm{AG}$, ratio $=1.47(\mathrm{t}(238) \mathrm{n}=2.53 ; \mathrm{p}<0.01)$. Taken together, the thresholds at $28.5 \mathrm{~cm}$ were higher than at $57 \mathrm{~cm}(\mathrm{~F}(1,476)=7.07 ; \mathrm{p}<0.01)$. The significant difference in thresholds at $28.5 \mathrm{~cm}$ and $57 \mathrm{~cm}$ again suggests that a separate depth threshold may operate at close viewing distances. Although the magnitude of the effect in this experiment was not quite as great as in experiment 1 (the average ratio for MFB and AG in experiment 1 was 1.6 , and here it was 1.4 ), it remained significantly above chance. The difference in effect sizes may be due to the fact that in experiment 2 only vergence and vertical disparities were varied, whereas in experiments 1 and 3 there were a range of cues to viewing distance available such as relative size of objects visible at different viewing distances and a texture gradient along the plane of the tabletop (e.g. Durgin et al, 1995; Glennerster et al, 1996; 1998). These may have increased the effectiveness of the changes in viewing distance to the visual system. In experiments 2 and 3 the controls introduced some degree of unnatural viewing into the situation, but the rise in thresholds at the closest viewing distance remained.

Figure 6 about here

\section{Discussion}

The results reported here show that thresholds to discriminate the structure in stereoscopic corrugations do not depend only on the disparity in the stimulus. The rise in 
thresholds at the close viewing distance is in the direction predicted if subjects were limited by a minimum perceived depth in addition to a minimum disparity threshold.

Although our results seem to contradict the conclusion of earlier studies (Ogle, 1958; Jameson and Hurvich, 1959), the data from those studies are in fact consistent with the framework we have set out here (see figure 1). This predicts that the effect of a minimum perceived depth would only be apparent at the closest viewing distances. Ogle (1958) and Jameson and Hurvich (1959) found higher thresholds than we did and they did not test the short viewing distance at which we found raised thresholds. On both counts, they would not have been expected to find a variation in thresholds if the model illustrated in figure 1 is correct.

It is reasonable to question why the visual system should have a separate threshold for perceived depth in addition to one for disparity detection. One suggestion is that a perceived depth limit could reflect the operation of a 'depth module' that has inputs from many modalities and has certain fixed properties, including a threshold for depth detection, that are independent of the modality of the input. Such a module might exist at a 'higher' stage in the visual system than the initial processing of disparity in V1. There is clear evidence of such a series of stages of disparity processing in visual cortex where the later stages are often assumed to provide representations that have properties closer to those of perceived depth than the types of representations based on local disparity computations characteristic of primary visual cortex, V1 (e.g. Cumming and Parker, 1997; Hibbard et al, 2000; Harris and Parker, 1995). For example, von der Heydt et al (2000) found that V1 cells were tuned to disparity, but showed that V2 cells responded to higher order features of the disparity field such as steps or edges that are more closely associated with physical features. Compelling 
evidence of the role that structures further along the visual pathway play in depth perception was provided by DeAngelis et al (1998) who showed that electrical stimulation of area V5 could bias perceptual judgments of awake behaving monkeys. A similar picture of stages in disparity processing has also emerged from human fMRI work. Such results show that cells in V3A may play a special role in stereoscopic acuity (Backus et al., 2001; Tsaso et al., 2003) and relative (V3, V7 and MT) and absolute (V4 and V8) disparities may be encoded in different cortical areas following joint processing through V1, V2 and V3 (Neri et al., 2004). There is also psychophysical evidence suggesting that a 'depth module' may exist where information from a range of cues may be combined into an 'amodal' representation which has certain fixed properties independent of the modality of the input. For example, threshold elevation and tilt and size aftereffects can transfer between different visual cues such as disparity, motion parallax and luminance (Rogers and Graham, 1984; Bradshaw \& Rogers, 1996; Nawrot \& Blake, 1991; Bradshaw, Hibbard \& Hogervorst, 1999). If such a depth module did exist then it could conceivably be associated with a separate noise process in addition to the noise that is present in the signals arriving from individual modalities.

An alternative explanation is that despite our efforts to ensure that the retinal stimulus was the same at different viewing distances, some 'low level' factor explains the rise in thresholds for the closest viewing distance. One possibility is that increased effort to maintain fixation at close viewing distances might increase thresholds, for example as a result of eye movement jitter.

Whichever of these very different types of explanation turns out to be correct, there must be a source of noise that is greater at $28.5 \mathrm{~cm}$ than at the other viewing distances we tested. The careful experimental controls across the three experiments presented were 
designed to eliminate any optical source of error that might have co-varied with changes in viewing distance. If we succeeded in this aim, then there must be another cause for the rise in thresholds at the closest viewing distance. We have suggested that there might be some internal source of noise that varies with the perceived depth of the stimulus. Thus, we conclude that performance in stereoscopic acuity tasks could be limited by the minimum perceived depth in a stimulus.

\section{Acknowledgements}

We thank Prof. BJ Rogers at the University of Oxford in whose laboratory the experiments were carried out and Tam Curnow for advice on statistical analysis. These results were reported in preliminary form at ARVO (Bradshaw, Rogers \& Glennerster, 1995). Supported by the Royal Society.

\section{References}

Backus BT, Fleet DJ, Parker AJ \& Heeger DJ, (2001) Human cortical activity correlates with stereoscopic depth perception. J. Neurophysiol. 86, $2054-2068$.

Bradshaw MF, Glennerster A, Rogers BJ (1996) The effect of display size on disparity scaling from differential perspective and vergence cues Vision Research 361255 - 1265.

Bradshaw MF, Hibbard PB, Hogervorst MA (1999) Tilt and size aftereffects in motion parallax, stereopsis and luminance. Investigative Ophthalmology and Visual Science. 40 S804 
Bradshaw MF, Parton AD, Eagle RA (1998) The interaction of binocular disparity and motion parallax in determining perceived depth and perceived size Perception. 271317 1333.

Bradshaw MF, Rogers BJ (1996) The combination of stereo and motion information Vision Research. 36 3457-3468.

Bradshaw MF, Rogers BJ, (1999) Sensitivity to stereoscopic corrugations as a function of corrugation frequency Vision Research 39 3049-3056.

Bradshaw MF, Rogers BJ, Glennerster A (1995) Are binocular disparity thresholds affected by viewing distance? Investigative Ophthalmology and Visual Science, 36230.

Cormack, R.H. (1984) Stereoscopic depth perception at far viewing distances. Perception \& Psychophysics, 35, 423-428.

Cumming B.G., Johnston E.J. \& Parker A.J. (1991) Vertical disparities and perception of three-dimensional shape. Nature, 349, 411-413.

Cumming BG \& Parker AJ (1997) Responses of primary visual cortical neurons to binocular disparity without depth perception. Nature, 389, 280-283.

DeAngelis GC, Cumming BG \& Newsome WT (1998) Cortical area MT and the perception of stereoscopic depth. Nature, 394, 677-680.

Durgin F H, Proffitt DR, Olson JT, Reinke KS (1995) Comparing depth from binocular disparity to depth from motion Journal of Experimental Psychology: Human Perception and Performance 21 679-99

Erkelens CJ. (2001) Organisation of signals involved in binocular perception and vergence control. Vision Research, 41, 3497-503

Finney, D.J. (1971). Probit Analysis. Cambridge University Press (3rd Edition). Foley, J.M. (1980) Binocular distance perception. Psychological Review, 87, 411-434. 
Frisby JP, Buckley D \& Duke, PA (1996) Evidence for the good recovery of lengths of real objects seen with natural stereo viewing. Perception, 25, 129-54.

Glennerster A, Rogers BJ, Bradshaw MF (1996) Stereoscopic depth constancy depends on the subject's task Vision Research, 36, 3441-3456.

Glennerster A, Rogers BJ, Bradshaw MF (1998) Cues to viewing distance for stereoscopic depth constancy Perception, 27,1357 - 1367.

Green, D.M. and Swets, J.A. (1966) Signal Detection Theory and Psychophysics, New York: John Wiley \& Sons, Inc., Chapter 9, pp 235-275

Harris, J.M. \& Parker, A.J. 1995 Independent neural mechanisms for bright and dark information in binocular stereopsis. Nature, 374, 808-811

Harris JM, Keeble DRT, Nisihida S (1998) Effects of viewing distance on stereoacuity, Panum's fusional limit and upper depth limit. Invest. Ophthalmology Vis Sci. 39 S623.

Hibbard PB, Bradshaw MF, Eagle RA (2000) Binocular disparity influences the maximum displacement limits of motion perception Proceedings of the Royal Society, B 2671369 1374

Jameson D., \& Hurvich L. (1959) Note on factors influencing the relation between stereoscopic acuity and observation distance. Journal of the Optical Society of America 49 639.

Judge S.J, Cumming B.G. (1986) Neurons in the monkey midbrain with activity related to vergence eye movement and accommodation. Journal of Neurophysiology, 55, 915-30

Johnston E.B. (1991) Systematic distortions of shape from stereopsis Vision Research 31 $1351-1360$

Kaufman L. (1974) Sight and Mind and Introduction to Visual Perception. Oxford University Press, New York. 
Nawrot, M. \& Blake, R. (1991) The interplay between stereopsis and structure from motion. Perception and Psychophysics, 49, 230-244.

Neri, P, Bridge H, \& Heeger, DJ (2004) Stereoscopic processing of absolute and relative disparity in human visual cortex. Journal of Neurophysiology 92, 1880-1891.

O’Leary A., Wallach H. (1980) Familiar size and linear perspective as distance cues in stereoscopic depth constancy. Perception and Psychophysics, 27, 131-135

Ogle K.N. (1958) Note on stereoscopic acuity and observation distance. Journal of the Optical Society of America 48 794-798.

Ono, H. and Comerford, J. (1977) Stereoscopic depth constancy. In Epstein, W, (Ed.) Stability and Constancy in Visual Perception: Mechanisms and Processes, Wiley, New York.

Predebon J. (1993) The familiar size cue to distance and stereoscopic depth perception. Perception, 22, 985-995.

Rogers B.J. \& Bradshaw M.F. (1993) Vertical disparities, differential perspective and binocular stereopsis. Nature, 36, 253-255.

Rogers B.J. \& Bradshaw M.F. (1995) Disparity scaling and the perception of fronto-parallel surfaces. Perception 24 155-179.

Rogers, B.J. \& Graham, M.E. (1983) Anisotropies in the perception of three-dimensional surfaces, Science, 221, 1409-1411.

Rogers, B.J. \& Graham, M.E. (1984). Aftereffects from motion parallax and stereoscopic depth: similarities and interactions. In L. Spillman, B.R. Wooten (Eds), Sensory Experience, Adaptation and Perception: Festschrift Ivo Kohler (pp 603-619) Hillsdale NJ: Lawrence Erlbaum Associates. 
Tittle J S, Todd J T, Perotti V J , Norman J F (1995) Systematic distortion of perceived three dimensional structure from motion and binocular stereopsis Journal of Experimental Psychology : Human Perception and Performance 21 663-78

Trotter Y., Celebrini S., Stricanne B., Thorpe S., and Imbert M, (1992) Modulation of neural stereoscopic processing in primate area V1 by the viewing distance. Science 257 12791281

Tsaso DY, Vanduffel W, \& Sasaki, Y (2003) Stereopsis activates V3A and caudal intraparietal areas in macques and humans. Neuron 39, 555-568.

von der Heydt R, Zhou H, Friedman HS. (2000) Representation of stereoscopic edges in monkey visual cortex. Vision Research, 40, 1955-1967.

Wallach, H. \& Zuckerman, C. (1963) The constancy of stereoscopic depth. American journal of Psychology, 76, 404-412. 


\section{Figure legends}

\section{Figure 1:}

Possible limits on depth perception. The disparity amplitude of the corrugated surface at threshold is plotted against viewing distance. If depth discrimination depended only on the disparity in the stimulus, then thresholds should be the same at all viewing distances (horizontal dotted line). If there was a minimum depth amplitude (fixed in mm) below which depth discrimination was not possible, then thresholds would vary according to the inverse squared relationship between disparity and distance $\left(1 / \mathrm{D}^{2}\right.$, dotted line with slope -2$)$. The hypothesis pursued in this paper is that for a corrugation to be detectable it must be greater than both a disparity and a depth threshold (solid line, see text). The effect of the minimum depth threshold would only be observed at near viewing distances.

\section{Figure 2:}

The angular sizes of the stimuli, corrugation frequency and size of random dots were kept constant at the different viewing distances.

\section{Figure 3:}

Results of Experiment 1. (a) Thresholds (mm) for MFB for the 5 modulation frequencies at the 3 real viewing distances tested. Horizontal dotted lines indicate predicted thresholds according to the inverse square law (separated by a factor of 4). (b) Thresholds (arc sec) for MFB for the 5 modulation frequencies at the 3 viewing distances tested. Error bars depict \pm SE.

\section{Figure 4:}

Thresholds (arc sec) plotted against real viewing distance $(\mathrm{cm})$ for the 4 subjects, for the modulation frequency at which thresholds were lowest $(0.4 \mathrm{cpd})$. Data for subject MFB are re-plotted from figure 3 . Error bars depict \pm SE. The dotted line in figures 4-6 indicates the slope of the threshold/distance function expected if thresholds were limited by a fixed minimum depth (see figure 1).

\section{Figure 5:}

Results of Experiment 2. Thresholds (arc sec) plotted against simulated viewing distance $(\mathrm{cm})$ for the 4 subjects $(0.4 \mathrm{cpd})$. Error bars depict \pm SE. Real viewing distance was $57 \mathrm{~cm}$.

\section{Figure 6:}

Results of Experiment 3. Thresholds (arc sec) plotted against real viewing distance $(\mathrm{cm})$ for the 4 subjects $(0.4 \mathrm{cpd})$. Error bars depict \pm SE. 


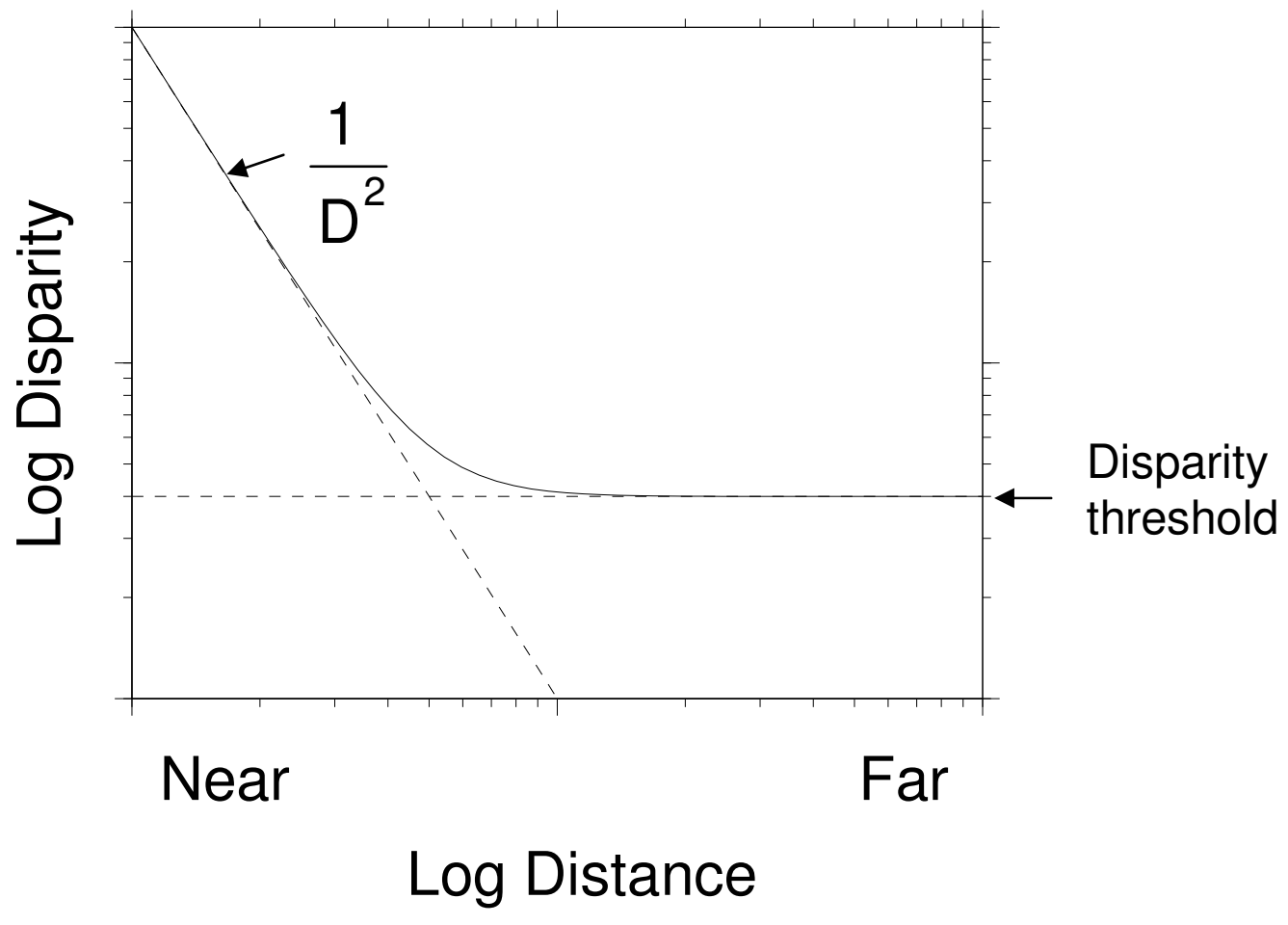

Bradshaw - Figure 1 


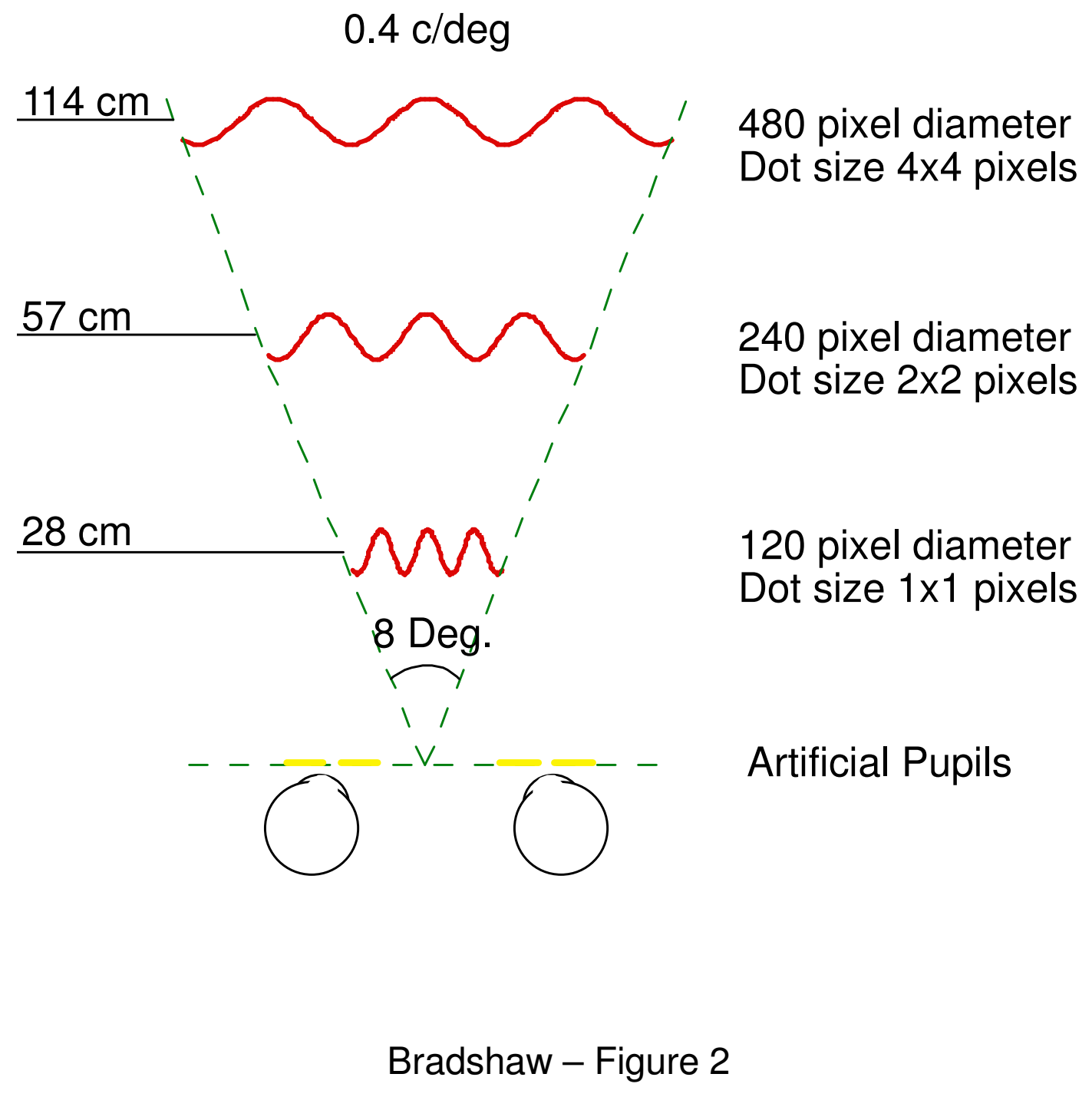


(a)

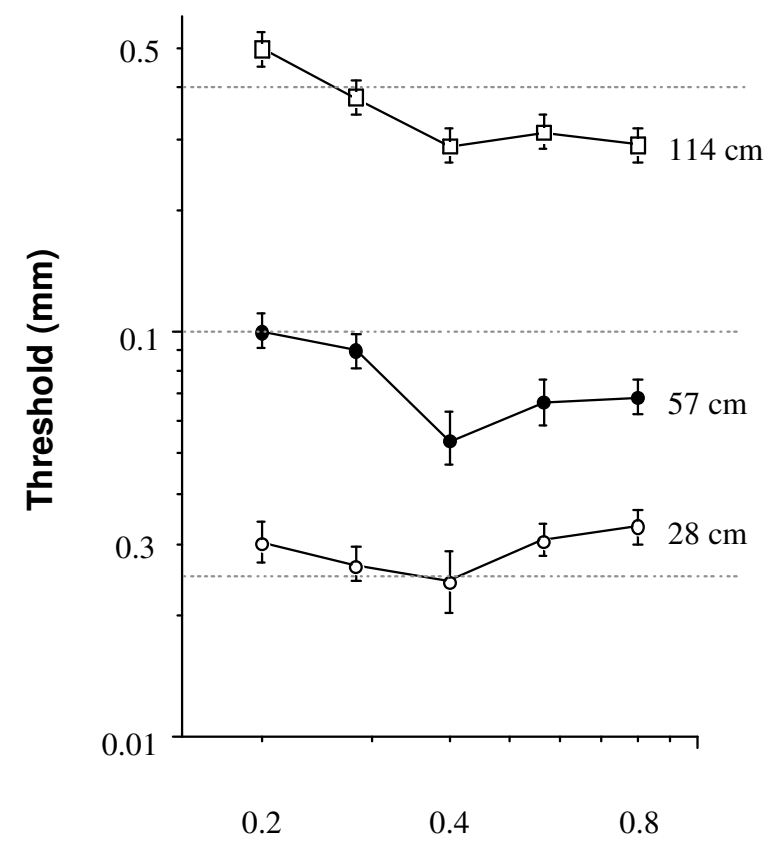

Spatial frequency (cycles/degree) (b)

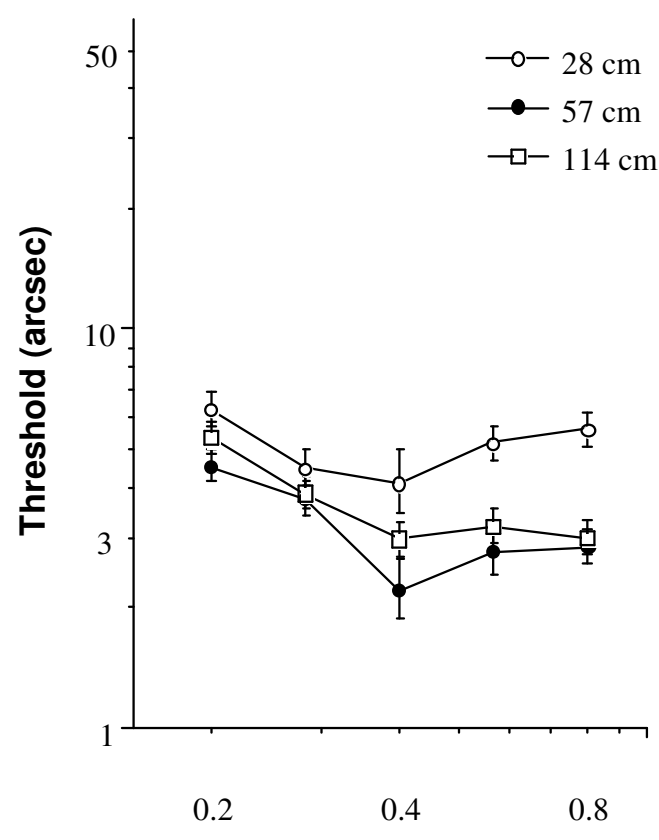

Spatial frequency (cycles/degree)

Bradshaw - Figure 3 


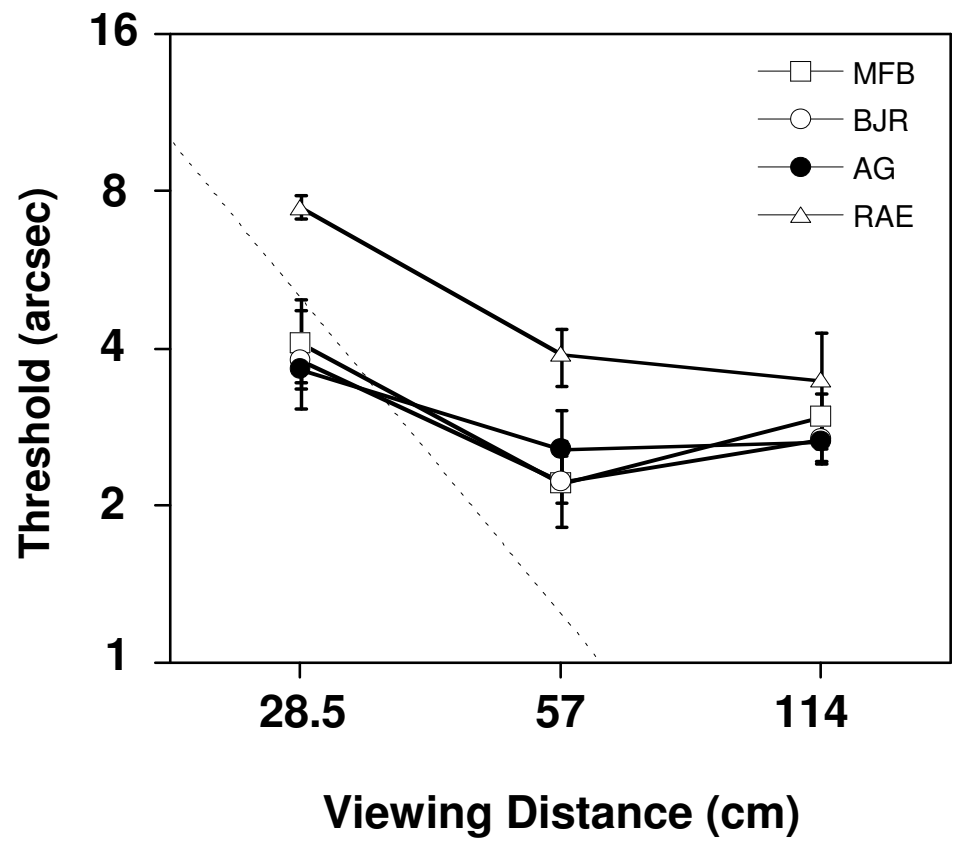

Bradshaw - Figure 4 


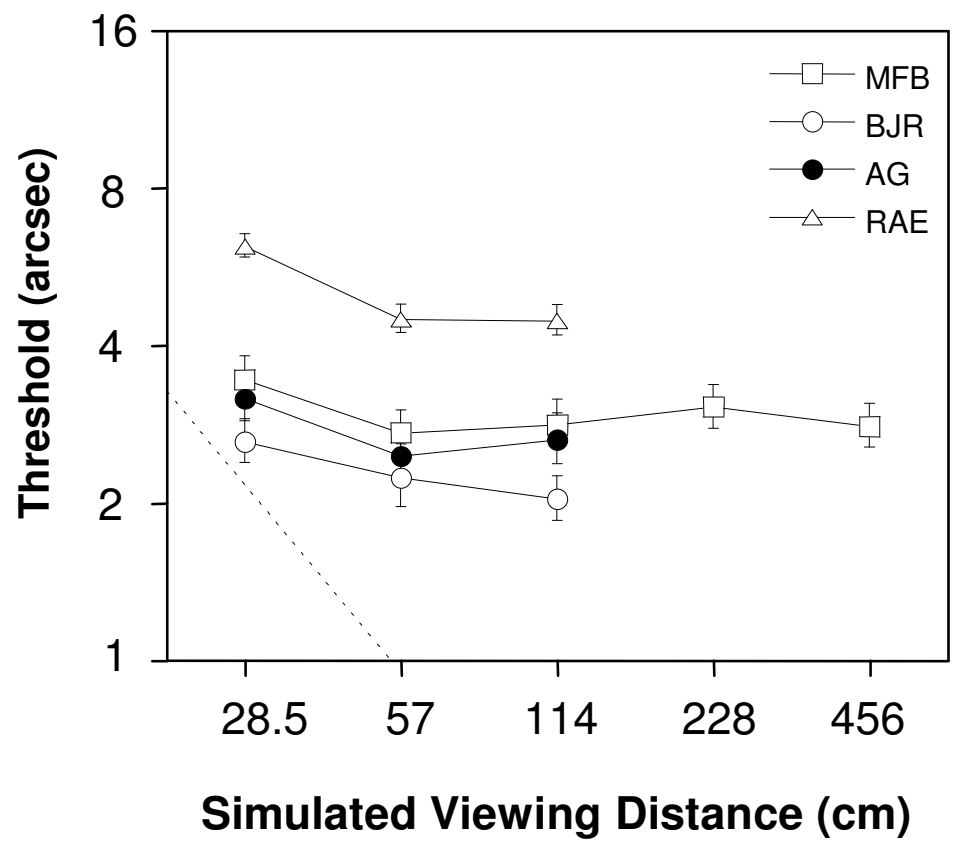

Bradshaw - Figure 5 


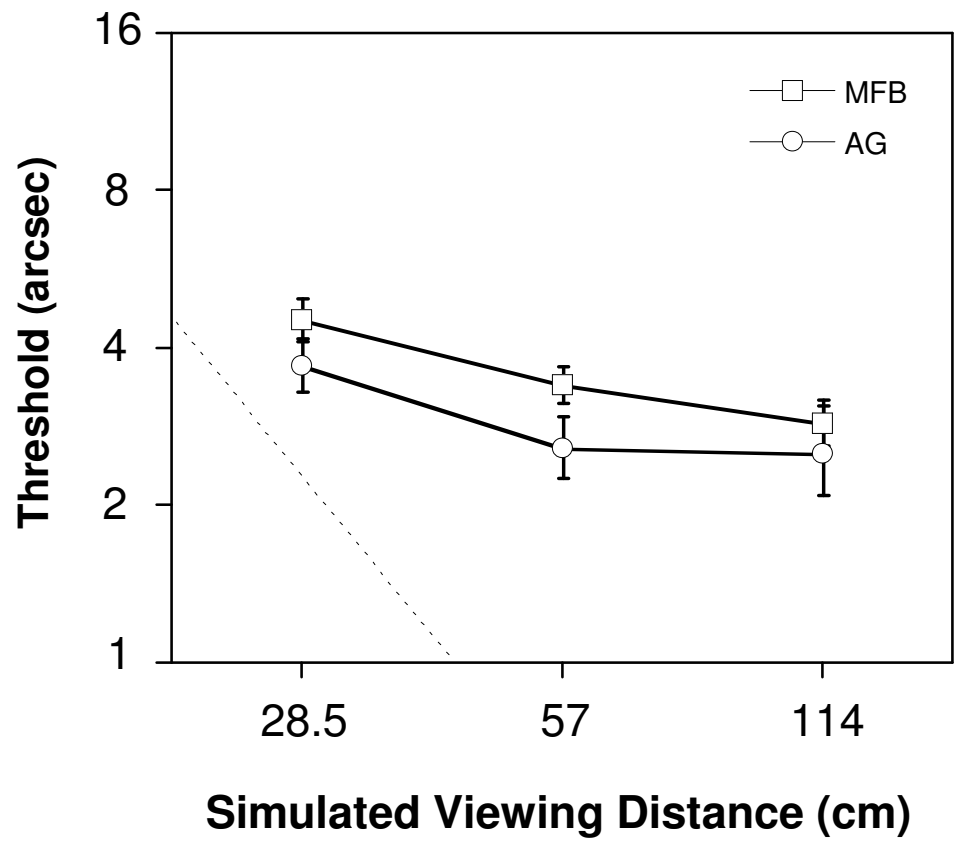

Bradshaw - Figure 6 